Occupational Safety and Health, Cincinnati, United States of America; ${ }^{4}$ University of Leeds, Leeds, United Kingdom; ${ }^{5}$ INRETS, Lyon, France; ${ }^{6}$ University of Oregon, Corvallis, United States of America; ${ }^{7}$ University of Ottawa, Ottawa, Canada; ${ }^{8}$ University of Montreal Hospital Research Centre, Montreal, Canada; ${ }^{9}$ Massey University, Wellington, New Zealand; ${ }^{10}$ INRS-Institut Armand Frappier, Montreal, Canada; ${ }^{11}$ Gertner Institute, Tel Aviv, Israel; ${ }^{12}$ DFKZ, Heidelberg, Germany; ${ }^{13}$ Institute of Occupational Medicine, Edinburgh, United Kingdom

10.1136/oemed-2013-101717.241

Objectives Brain tumours are a serious, often fatal disease with few established risk factors. Although ionising radiation has been clearly linked with brain tumours, there are a number of other environmental and occupational agents suspected including extremely low frequency magnetic field (ELF-MF) exposure. However the literature is inconsistent, and questions remain due to small sample sizes and limitations in exposure assessment in previous studies. The objective of this paper was to examine the association between occupational exposure to ELF-MF in different time windows and brain tumours in the large-scale INTEROCC study.

Methods The INTEROCC study is formed by seven participating countries Australia, Canada, France, Germany, Israel, New Zealand, United Kingdom) from the parent INTERPHONE study. Cases of primary brain glioma and meningioma aged at least 20 years were recruited between 2000 and 2004. Detailed occupational history data was collected for jobs held at least six months. Job titles were coded into standard international occupational classifications and estimates of mean workday ELF-MF exposure assigned based on a job exposure matrix. Conditional logistic regression was used to obtain adjusted odds ratios and 95\% confidence intervals.

Results Data on a total of 3,978 brain tumour cases, including 2,054 gliomas and 1,924 meningiomas, were analysed with 5,601 control subjects. Estimates of cumulative exposure, time-weighted average exposure, maximum exposure, and exposure duration were calculated for exposure 1-4, 5-9, and $10+$ years in the past. Estimates of mean cumulative exposure were higher for males, older participants, and participants with lower levels of educational attainment. Positive associations between different indicators of ELF-MF exposure in the 1-4 year time window and glioma but not meningioma were observed.

Conclusion Occupational ELF-MF exposure may play a role in the promotion of glioma, however findings may also be due to reverse causality or other methodological sources of bias.

\section{QUANTITATIVE MEASUREMENTS OF OCCUPATIONAL EXPOSURE TO STATIC MAGNETIC STRAY FIELDS FROM MRI SCANNERS IN CLINICAL AND RESEARCH ENVIRONMENTS}

${ }^{1} \mathrm{~K}$ Schaap, ${ }^{2}$ Cambron - Goulet, ${ }^{1}$ Christopher - De Vries, ${ }^{1}$ Kromhout. ${ }^{1}$ Utrecht University, Utrecht, The Netherlands; ${ }^{2}$ Université de Sherbrooke, Sherbrooke (Québec), Canada

10.1136/oemed-2013-101717.242

Objectives Workers' exposure to static magnetic stray fields from MRI scanners was evaluated in a large cross-sectional study in The Netherlands. Factors determining the exposure to static magnetic fields (SMF) and time-varying magnetic fields (TVMF) like scanner characteristics, work patterns and individual practices could be quantified.

Methods Occupational exposure to static magnetic fields (B) and rate of change of field due to movement through a static magnetic field $(\mathrm{d} B / \mathrm{d} t)$ were measured using a Magnetic Field Dosimeter (University of Queensland). About 480 predominantly full-shift measurements were collected from more than 300 employees working at 14 clinical or research MRI facilities. During measurement days, participants kept a log of the tasks they performed and the scanner (s) at which they worked.

Results Highest peak $\mathrm{B}$ and $\mathrm{d} B / \mathrm{d} t$ exposures were observed among MRI radiographers and research staff. Peak exposures were highest in academic hospitals (mean [range]: $814 \mathrm{mT}$ [364928]; $1291 \mathrm{mT} / \mathrm{s}$ [27 - 5057]) and lowest for people working in experimental animal imaging facilities (mean [range]: $227 \mathrm{mT}$ [31 - 625]; $395 \mathrm{mT} / \mathrm{s}$ [32 - 1329]). Scanner field strength showed a strong association with peak $B$ and $\mathrm{dB} / \mathrm{dt}$ exposure when subjects worked near a closed bore scanner. However, for small bore scanners this association appeared to be negative. This could be partially explained by variability in shielding (active vs. passive) of the small-bore magnets.

Conclusions For assessment of exposure for epidemiological studies classification solely based on scanner field strength is insufficient. The type of scanner (open/closed/extremity scanner; large/small bore) and type of shielding of the static magnetic field (active/passive) should be taken into account as well.

\section{OCCUPATIONAL EXTREMELY LOW FREQUENCY MAGNETIC FIELD EXPOSURE AND CANCER INCIDENCE IN A LARGE PROSPECTIVE COHORT STUDY}

${ }^{1} \mathrm{~T}$ Koeman, ${ }^{2}$ Leo, ${ }^{1}$ Slottje, ${ }^{1}$ Kromhout, ${ }^{3}$ Bausch-Goldbohm, ${ }^{2}$ van der Brandt, 'Vermeulen. ${ }^{1}$ IRAS, Utrecht, Nederland; ' 2 Maastricht University, Maastricht, Nederland; ${ }^{3} T N O$, Leiden, Nederland

\subsection{6/oemed-2013-101717.243}

Objectives This study investigated the association between exposure to occupational extremely low frequency magnetic fields (ELF-MF) and various types of cancer within the prospective Netherlands Cohort Study (NLCS).

Methods For this case-cohort analysis, 120,852 men and women aged 55 to 69 years at time of enrollment in 1986 were followed up (17.3 years) for incident cases of lung, breast, brain and haematopoietic cancers and their subtypes. Information on occupational history and potential confounders such as sex, age, smoking, alcohol use and attained educational level were collected at baseline through a self-administered questionnaire.

Occupations were coded using the International Standard Classification of Occupations (ISCO-88). Occupational ELFMF exposure was assigned by using a semi-quantitative ELF job-exposure matrix which assigns ordinal exposure levels (background, low and high exposure) based on intensity and probability of exposure. Metrics of ELF-MF exposure were ever low and ever high exposure versus background exposure, duration of exposure, and cumulative exposure to ELF-MF up to baseline. Associations with cancer incidence were analysed with Cox-regression using attained age as underlying time scale.

Results Ever low or ever high exposure to ELF-MF showed no effect on cancer incidence of lung, breast, brain cancer, nor any of the assessed subtypes. Duration and cumulative exposure also showed no effect of ELF-MF exposure on these cancer sites. Ever high exposed to ELF-MF showed a significant association with acute myeloid leukaemia (AML) (hazard ratio [HR] 2.09; 95\% confidence interval [CI] 1.05-4.15) and follicular lymphoma (HR 2.40; 95\%CI 1.00 - 5.77). In addition, cumulative 